\title{
The methylated N-terminal tail of RCC1 is required for stabilisation of its interaction with chromatin by Ran in live cells
}

\author{
Ekarat Hitakomate1, Fiona E Hood ${ }^{1}, 2$, Helen S Sanderson ${ }^{1}$ and Paul R Clarke*1
}

\begin{abstract}
Background: Regulator of chromosome condensation 1 (RCC1) is the guanine nucleotide exchange factor for Ran GTPase. Localised generation of Ran-GTP by RCC1 on chromatin is critical for nucleocytoplasmic transport, mitotic spindle assembly and nuclear envelope formation. Both the N-terminal tail of RCC1 and its association with Ran are important for its interaction with chromatin in cells. In vitro, the association of Ran with RCC1 induces a conformational change in the N-terminal tail that promotes its interaction with DNA.

Results: We have investigated the mechanism of the dynamic interaction of the a isoform of human RCC1 (RCC1a) with chromatin in live cells using fluorescence recovery after photobleaching (FRAP) of green fluorescent protein (GFP) fusions. We show that the N-terminal tail stabilises the interaction of RCC1 a with chromatin and this function can be partially replaced by another lysine-rich nuclear localisation signal. Removal of the tail prevents the interaction of RCC1 a with chromatin from being stabilised by Ran ${ }^{T 24 N}$, a mutant that binds stably to RCC 1 a. The interaction of RCC1a with chromatin is destabilised by mutation of lysine $4(\mathrm{~K} 4 \mathrm{Q})$, which abolishes a-N-terminal methylation, and this interaction is no longer stabilised by Ran ${ }^{T 24 N}$. However, a-N-terminal methylation of RCC1 a is not regulated by the binding of Ran ${ }^{T 24 N}$. Conversely, the association of Ran with precipitated RCC1 a does not require the N-terminal tail of RCC1a or its methylation. The mobility of RCC1 a on chromatin is increased by mutation of aspartate 182 (D182A), which inhibits guanine-nucleotide exchange activity, but RCC1 aD182A can still bind nucleotide-free Ran and its interaction with chromatin is stabilised by Ran ${ }^{\mathrm{T} 24 \mathrm{~N}}$.

Conclusions: These results show that the stabilisation of the dynamic interaction of RCC 1 a with chromatin by Ran in live cells requires the N-terminal tail of RCC1a. a-N-methylation is not regulated by formation of the binary complex with Ran, but it promotes chromatin binding through the tail. This work supports a model in which the association of $\mathrm{RCC} 1 \mathrm{a}$ with chromatin is promoted by a conformational change in the a-N-terminal methylated tail that is induced allosterically in the binary complex with Ran.
\end{abstract}

\section{Background}

The small Ran GTPase plays key roles during the cell cycle in eukaryotic cells [1]. Generation of RanGTP from RanGDP requires a Ran guanine nucleotide exchange factor (RanGEF) known as Regulator of Chromosome Condensation 1 (RCC1) in vertebrates [2,3]. RCC1 is localised predominantly to chromatin throughout the cell cycle $[4,5]$. Hydrolysis of GTP to GDP by Ran is greatly stimu-

\footnotetext{
* Correspondence: p.r.clarke@dundee.ac.uk

1 Biomedical Research Institute, School of Medicine, College of Medicine, Dentistry and Nursing, University of Dundee, Ninewells Hospital and Medical School, Dundee DD1 9SY, UK

Full list of author information is available at the end of the article
}

lated by Ran GTPase-activating protein (RanGAP) in the cytoplasm [6]. The distinct localisation of these regulators results in a high concentration of RanGTP relative to that of RanGDP in the vicinity of chromatin [7]. Within the nucleus, RanGTP promotes the assembly of export complexes between proteins carrying a leucine-rich nuclear export signal (NES) and exportin (Crm1), while causing the disassembly of imported complexes formed between proteins carrying a lysine-rich nuclear import signal (NLS) and importins. Thus, RanGTP determines the direction of nucleocytoplasmic transport during interphase [8]. In animal cells in which the nuclear enve- 
lope breaks down during mitosis and the separation of the nucleoplasm and cytoplasm is lost, continued generation of RanGTP on chromosomes by RCC1 is thought to provide a spatial signal to organise spindle assembly [9]. Localised generation of RanGTP by RCC1 on chromatin is therefore critical for the function of the Ran system throughout the cell cycle [1].

RCC1 has a core domain with a 7-bladed propeller structure [10] that interacts on one face with Ran [11] and is proposed to interact on the other face with chromatin $[12,13]$, possibly through core histones $\mathrm{H} 2 \mathrm{~A}$ and $\mathrm{H} 2 \mathrm{~B}$ [14]. Near to the $\mathrm{N}$-terminus is a short flexible region that contains a functional lysine-rich nuclear localisation signal (NLS) that associates with the import receptor dimer formed by importin- $\alpha 3$ and importin- $\beta[5,15,16]$. In vitro, this basic N-terminal region (NTR) or tail can interact directly with DNA $[13,17]$ and in cells it is involved in both the concentration of RCC1 in the nucleus and in its interaction with chromatin [5]. RCC1 is modified in cells by removal of the initial $\mathrm{N}$-terminal methionine and mono-, di- or tri-methylation of the $\alpha$-amino group of the new $\mathrm{N}$-terminal residue (serine 2 in human $\mathrm{RCC} 1$ ). This modification is present throughout the cell cycle and promotes the localisation of RCC1 to mitotic chromosomes [18]. During mitosis, phosphorylation of RCC1 at serine 2 and serine 11 by CDK1-cyclin B1 dissociates RCC1 from importin- $\alpha 3$-importin- $\beta$ and regulates its interaction with chromatin $[19,20]$. In mammalian cells, RCC1 exists in at least three isoforms ( $\alpha, \beta$ and $\gamma$ ), which are probably generated by alternative splicing of the mRNA. RCC1 $\beta$ and $\mathrm{RCC} 1 \gamma$ have unique inserts after residue 24 which alter the length of their N-terminal tails. In the case of RCC1 $\gamma$, a 17 amino acid insert stabilises its interaction with chromatin, reduces importin binding and alters its regulation by phosphorylation at serine 11 [21].

Studies using RCC1 fused to green fluorescent protein (GFP) have shown that its interaction with chromatin in live cells is highly dynamic $[19,22,23]$. The rate of fluorescence recovery after photobleaching (FRAP) on chromatin is regulated by the association of GFP-RCC $1 \alpha$ with Ran [19,22]. Mutation of aspartate 182 of RCC1 $\alpha$ (D182A), which inhibits its guanine nucleotide exchange activity, destabilises the interaction of its GFP fusion with chromatin $[5,19]$. Conversely, co-expression of $\operatorname{Ran}^{\mathrm{T} 24 \mathrm{~N}}$, a mutant defective in nucleotide binding that forms a stable complex with RCC1 and inhibits its guanine nucleotide exchange activity $[24,25]$, strongly stabilises the interaction of GFP-RCC1 $\alpha$ with chromatin [22]. GFP-Ran ${ }^{\mathrm{T} 24 \mathrm{~N}}$ associates stably with chromatin throughout the cell cycle and co-localises with RCC1, consistent with formation of a stable binary complex with RCC1 [26]. Li et al. [22] proposed that the association of RCC1 with nucleotide-free Ran (apoRan) in a transient binary complex stabilises its interaction with chromatin, thereby linking the interac- tion of RCC1 with chromatin to generation of Ran-GTP. More recently, Hao and Macara [13] have developed a fluorescence resonance energy transfer (FRET)-based reporter in which $\mathrm{RCC} 1 \alpha$ was fused to CFP at its $\mathrm{N}$-terminal and YFP at its C-terminal (CFP-RCC1 $\alpha$-YFP). They showed that binding of $\operatorname{Ran}^{\mathrm{T} 24 \mathrm{~N}}$ or apoRan ${ }^{\mathrm{WT}}$ to CFPRCC $1 \alpha-Y F P$ caused a conformational change in the $\mathrm{N}$ terminal tail, which stabilised the interaction of CFPRCC1 $\alpha$-YFP with DNA in vitro and with chromatin in permeabilised cells. These authors proposed a model in which the interaction of Ran with RCC1 allosterically induces a conformational change in the $\mathrm{N}$-terminal tail, which then interacts with DNA and thereby stabilises the association of RCC1 with chromatin. In these experiments, however, the role of $\alpha-\mathrm{N}$-methylation, which was by necessity abolished in the CFP-RCC1 $\alpha$-YFP reporter, could not be tested. The role of the $\mathrm{N}$-terminal tail in the interaction of the RCC1-Ran binary complex with chromatin also remained to be demonstrated in vivo.

Here, we have tested the mechanism of the interaction of RCC $1 \alpha$ with chromatin in live cells using FRAP of GFP fusions. We show that the $\alpha-\mathrm{N}$-methylated tail of RCC1 $\alpha$ is important for the stability of the interaction of $\mathrm{RCC} 1 \alpha$ with interphase chromatin. Indeed, the methylated tail is required for the stabilising effect of $\operatorname{Ran}^{\mathrm{T} 24 \mathrm{~N}}$. These results provide strong support for an allosteric model of the interaction of the RCC1-Ran binary complex with chromatin in vivo.

\section{Results}

The N-terminal tail of RCC1 is required for stable association with interphase chromatin

To examine the role of the $\mathrm{N}$-terminal region (NTR) or tail of $\mathrm{RCC} 1 \alpha$ in its dynamic interaction with chromatin in live cells, we made $\mathrm{N}$-terminal and $\mathrm{C}$-terminal GFP fusion constructs of the RCC1 $\alpha \mathrm{N}$-terminal tail (residues $1-27)$, the RCC1 core domain $(\triangle 27 R C C 1)$ and full-length RCC1 1 . We also fused the RCC1 core domain with a classical lysine-rich monopartite nuclear localisation signal (PKKKRK) derived from SV40 T antigen (SV40 NLS) to generate SV40 NLS- 27 RCC1 (Figure 1A). When expressed in HeLa cells, this GFP fusion construct was concentrated in nuclei like full length RCC1 $\alpha$ (Figure 1B). GFP proteins expressed from the same vectors as the $\mathrm{N}$ terminal and $\mathrm{C}$-terminal tagged proteins (N-term-GFP and C-term-GFP, respectively) were used as controls. To monitor the dynamic interaction between RCC1 with chromatin, we performed fluorescent recovery after photobleaching (FRAP) experiments on nuclei in live HeLa cells expressing the GFP fusion constructs (Figure 1C).

As expected, N-term-GFP alone freely diffused in nuclei with half-time $\left(\mathrm{t}_{1 / 2}\right)$ of $0.15 \pm 0.11 \mathrm{~s}(\mathrm{n}=33)$ (Figure 1D) (See Additional file 1 Figure $S 1$ for original data). The FRAP half-time of GFP-RCC1 $\alpha$ was $0.84 \pm 0.23 \mathrm{~s}$ ( $\mathrm{n}=$ 


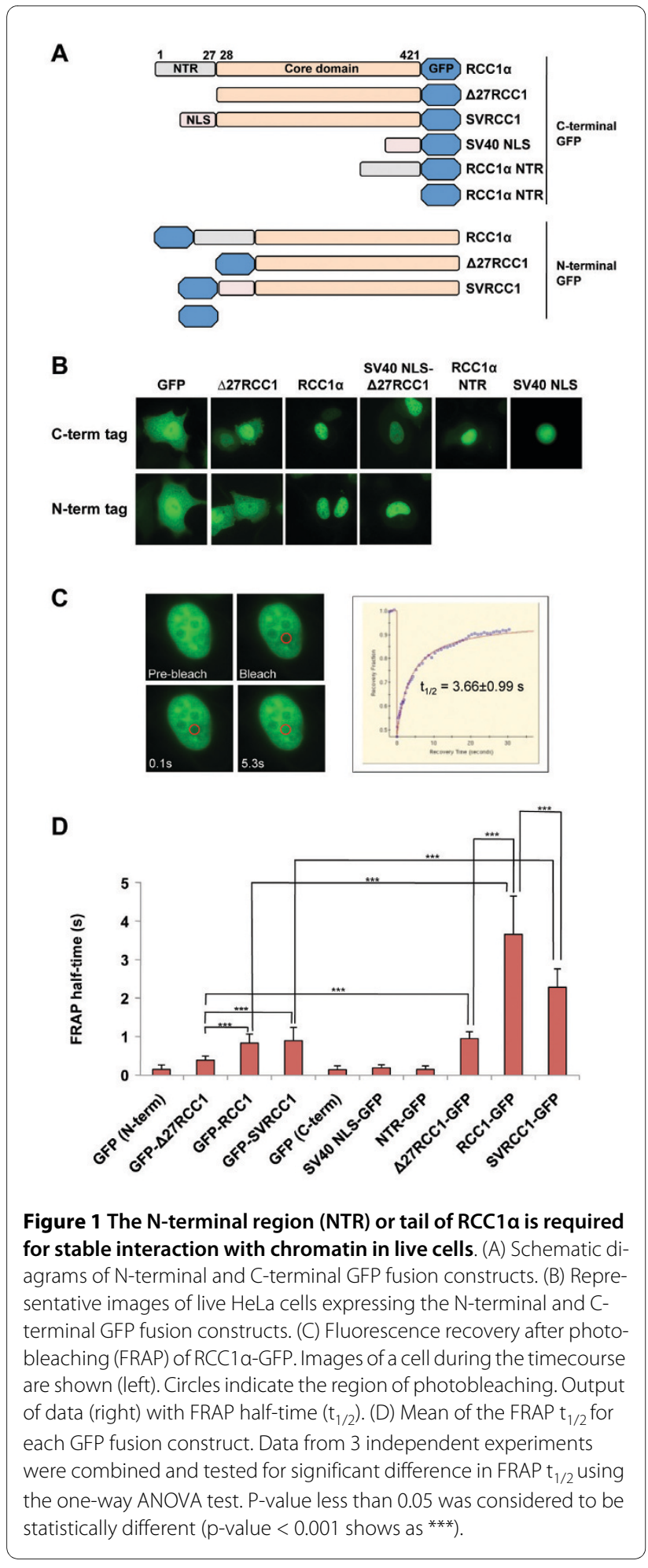

114) whereas $t_{1 / 2}$ of GFP- $\Delta 27 R C C 1$ was decreased to 0.39 $\pm 0.11 \mathrm{~s}(\mathrm{n}=48)$, consistent with a stabilising role for the $\mathrm{N}$-terminal tail in the interaction of RCC1 $\alpha$ with interphase chromatin [19]. However, GFP-SV40 NLS$\triangle 27 R C C 1$ was as mobile as the full-length RCC1 $\alpha$ with the FRAP $\mathrm{t}_{1 / 2}$ of $0.90 \pm 0.34 \mathrm{~s}(\mathrm{n}=96)$. These data indicate that, in the context of the $\mathrm{N}$-terminal fusion with GFP, which cannot be $\alpha-\mathrm{N}$-methylated, the positively charged residues in the NLS of RCC1 $\alpha$ weakly stabilise its interaction with chromatin. When the GFP is fused to the N-terminus, the weak stabilising effect is not specific to the tail of $\mathrm{RCC} 1 \alpha$ and it can be replaced by another lysine-rich NLS-containing sequence.

When full-length RCC1 $\alpha$ fused to GFP at its C-terminus was monitored it exhibited a much more stable association with chromatin than the N-terminal GFP tagged fusion protein, with a FRAP $\mathrm{t}_{1 / 2}$ of $3.66 \pm 0.99 \mathrm{~s}(\mathrm{n}=79)$ (Figure 1D). $\triangle 27 \mathrm{RCC1}$-GFP was considerably less stable $(0.95 \pm 0.18 \mathrm{~s} ., \mathrm{n}=23)$ than $\mathrm{RCC} 1 \alpha$-GFP, but was still significantly more stable than GFP- $\triangle 27 \mathrm{RCC} 1$. The FRAP $\mathrm{t}_{1 /}$ ${ }_{2}$ of SV40 NLS- $\triangle 27 R C C 1-G F P$ was $2.29 \pm 0.48$ s. $(\mathrm{n}=29)$, which was intermediate between $\triangle 27 R C C 1$-GFP and RCC1 $\alpha$-GFP. Thus, the free $\mathrm{N}$-terminal tail of RCC1 $\alpha$ stabilises the association of protein with interphase chromatin in live cells. This stabilising effect can be partially reproduced by another basic NLS sequence.

To test whether the $\mathrm{N}$-terminal tail alone is sufficient to interact with chromatin, the mobility of the isolated NTR-GFP was monitored. The FRAP $t_{1 / 2}$ of the isolated NTR-GFP $(0.15 \pm 0.09 \mathrm{~s}, \mathrm{n}=82)$ was as mobile as that of the GFP alone $(0.15 \pm 0.10 \mathrm{~s}, \mathrm{n}=54)$. Similarly, the monopartite SV40 NLS-GFP was nuclear and the FRAP $\mathrm{t}_{1 / 2}(0.19 \pm 0.08 \mathrm{~s}, \mathrm{n}=60)$ was not different from either GFP alone or NTR-GFP. Thus, the NLS-containing Nterminal tail of RCC1 $\alpha$ is insufficient to stabilise interaction with chromatin unless it is joined to the core domain.

\section{Stable Ran binding to $\mathrm{RCC} 1$ regulates the dynamic interaction of RCC1 with chromatin in an $\mathrm{N}$-terminal tail- dependent manner in live cells}

We next confirmed that the binding of $\operatorname{Ran}^{\mathrm{T} 24 \mathrm{~N}}$ to $\mathrm{RCC} 1 \alpha$ stabilises the interaction of RCC1 $\alpha$ with chromatin in live human cells. U2OS cells were transiently co-transfected with GFP alone or RCC1 $\alpha$-GFP together with the fluorescent protein mCherry alone, mCherry fused to wild-type Ran (mCherry-RanWT) or mCherry fused to $\operatorname{Ran}^{\mathrm{T} 24 \mathrm{~N}}$ (mCherry-Ran ${ }^{\mathrm{T} 24 \mathrm{~N}}$ ). As expected, the FRAP $\mathrm{t}_{1 / 2}$ of RCC $1 \alpha$-GFP in cells co-expressing mCherry-Ran ${ }^{\mathrm{T} 24 \mathrm{~N}}$ was significantly increased compared to that of RCC1 $\alpha$-GFP in cells co-expressing either mCherry or mCherry-Ran WT (Figure 2A, Figure 3, Table 1). In addition, the mobile fraction of RCC1 $\alpha$-GFP, calculated as the proportion of the initial fluorescent signal that is recovered after photobleaching, was significantly reduced in cells in which mCherry-Ran ${ }^{\mathrm{T} 24 \mathrm{~N}}$ was co-expressed with $\mathrm{RCC} 1 \alpha$-GFP compared to cells co-expressing either mCherry or mCherry-Ran ${ }^{W T}$ (Table 1). Therefore, $\operatorname{Ran}^{\mathrm{T} 24 \mathrm{~N}}$ specifi- 
A

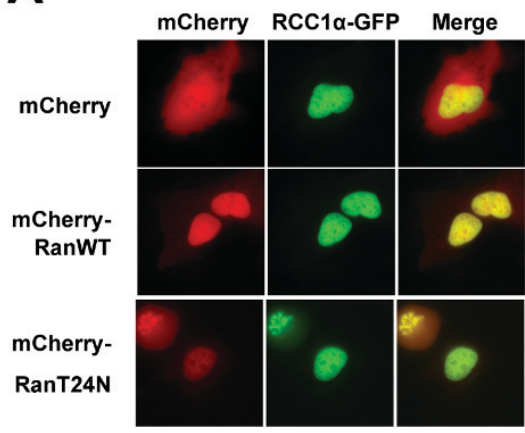

B

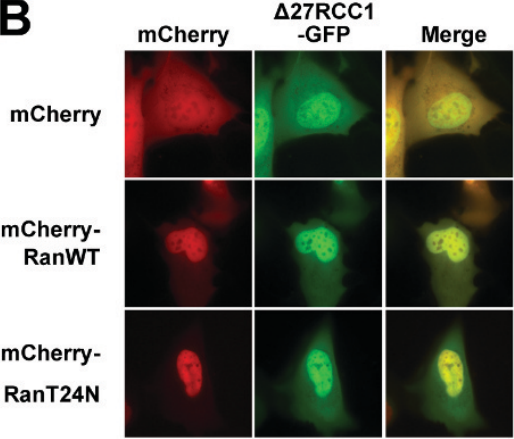

C

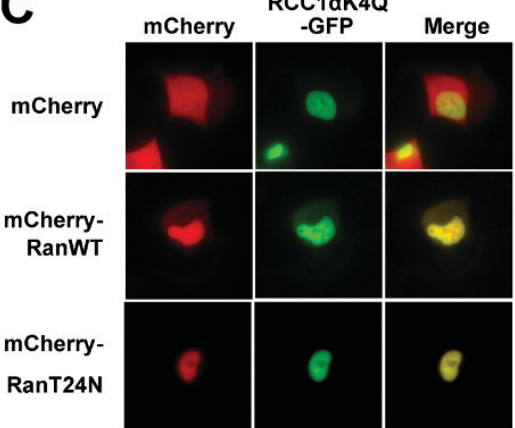

D

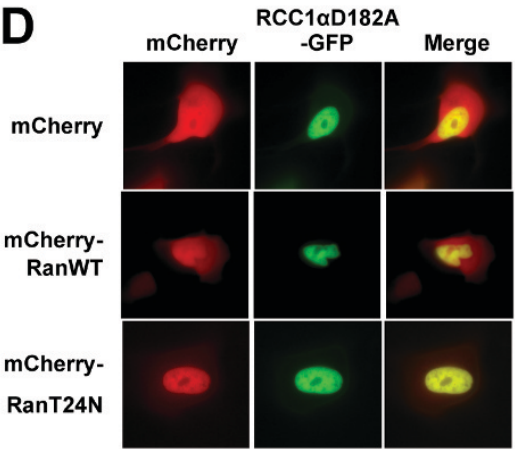

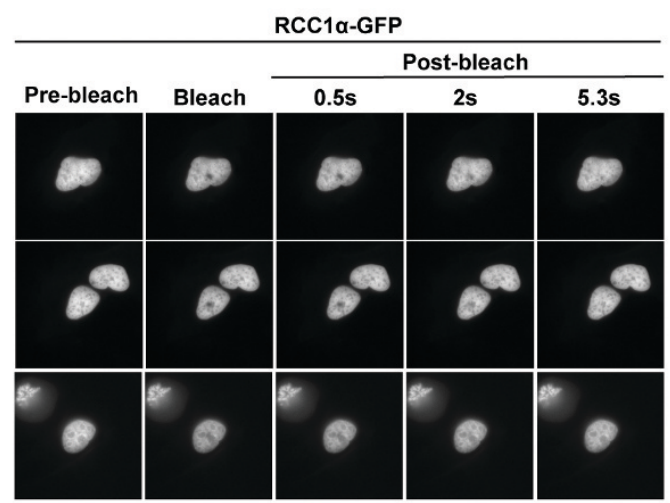

$\triangle 27 R C C 1-G F P$

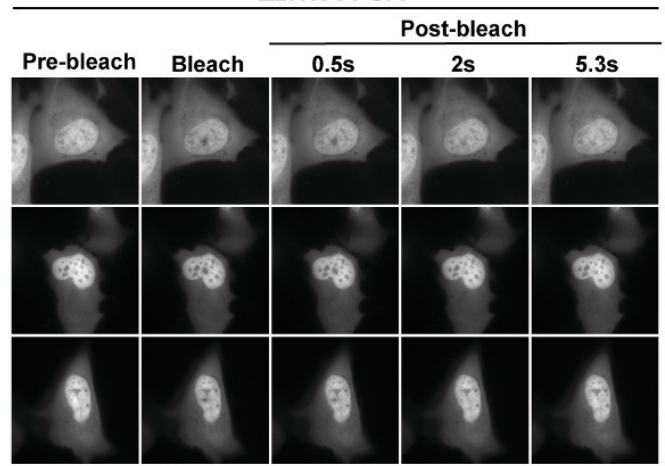

RCC1aK4Q-GFP

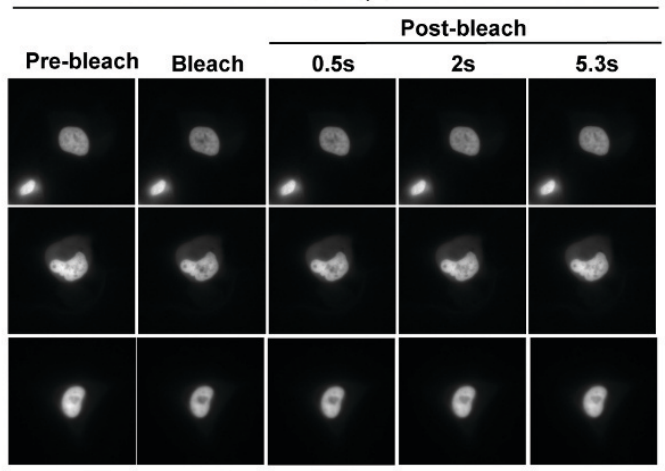

RCC1 12182A-GFP

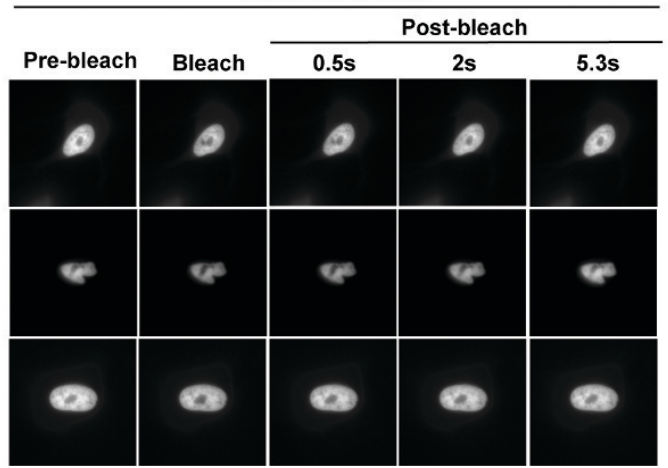

Figure 2 Effect of Ran on the interaction of RCC1 a with chromatin in live cells. Representative images of live U2OS cells co-expressing wild-type RCC1a-GFP (A), $\triangle 27$ RCC1-GFP (B), RCC1 a K4Q-GFP (C) or RCC1 a 182A_GFP (D) with mCherry empty vector (top), mCherry-RanWT (middle) or mCherryRan $^{T 24 N}$ (bottom) during fluorescence recovery after photobleaching (FRAP). 


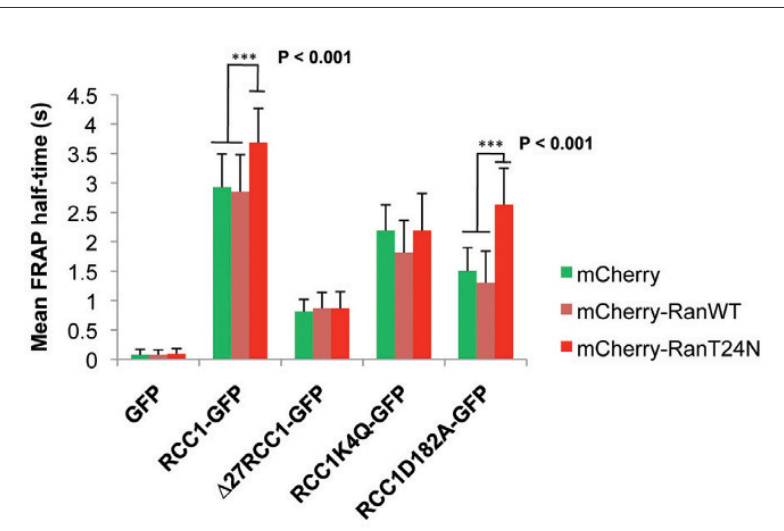

Figure 3 Stabilisation of the interaction of RCC 1 a with chromatin by Ran requires the methylated N-terminal tail of RCC1a. Bar chart showing the mean FRAP $t_{1 / 2}+$-SD of live U2OS cells co-expressing

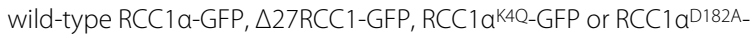
GFP with mCherry empty vector (green), mCherry-RanWT (pink) or $m C h e r r y-\operatorname{Ran}^{\top 24 \mathrm{~N}}$ (red). Data were tested for significant difference in FRAP $t_{1 / 2}$ using the Student's $t$-test. P-values considered to be statistically different $(<0.001)$ are indicated as ${ }^{* * *}$.

cally stabilises the interaction of $\mathrm{RCC} 1 \alpha$ with chromatin in live cells.
To test whether stabilization of interaction of $\mathrm{RCC} 1 \alpha$ with chromatin by $\operatorname{Ran}^{\mathrm{T} 24 \mathrm{~N}}$ requires the $\mathrm{N}$-terminal tail of RCC $1 \alpha$, we co-expressed a series of RCC1 $\alpha$-GFP fusion proteins (C-term GFP tagged) with mCherry alone, mCherry-Ran ${ }^{\mathrm{WT}}$ and mCherry-Ran ${ }^{\mathrm{T} 24 \mathrm{~N}}$ in U2OS cells. As expected, RCC1 $\alpha$-GFP localized to the nucleus, as did $\mathrm{RCC} 1 \alpha^{\mathrm{K} 4 \mathrm{Q}}-\mathrm{GFP}$ and $\mathrm{RCC} 1 \alpha^{\mathrm{D} 182 \mathrm{~A}}-\mathrm{GFP}$, whereas $\triangle 27 R C C 1-G F P$ was also present in the cytoplasm (Figure 2, left panels). FRAP experiments on these GFP fusions (Figure 2, right panels) showed that the FRAP $t_{1 / 2}$ of RCC $1 \alpha$-GFP was significantly increased when mCherry$\operatorname{Ran}^{\mathrm{T} 24 \mathrm{~N}}$ was co-expressed compared to when mCherry or mCherry-Ran ${ }^{W T}$ were co-expressed (Table 1; Figure 3). The truncated version of RCC1 lacking the N-terminal tail $(\triangle 27 R C C 1-G F P)$ showed a dramatic decrease in the FRAP $t_{1 / 2}(0.81 \pm 0.21 \mathrm{~s}$.) compared to the full-length protein $(\mathrm{RCC} 1 \alpha-\mathrm{GFP})(2.93 \pm 0.56 \mathrm{~s})$. However, $\Delta 27 \mathrm{RCC} 1-$ GFP failed to interact more stably in the presence of mCherry- $\operatorname{Ran}^{\mathrm{T} 24 \mathrm{~N}}(0.87 \pm 0.28 \mathrm{~s})$. These results show that the binding of Ran to RCC $1 \alpha$ stabilises the interaction of RCC $1 \alpha$ with chromatin in an N-terminal tail-dependent manner in live cells. Consistent results were also found using N-terminal GFP fusions of $\mathrm{RCC} 1 \alpha$, although the proteins were all more dynamic (less stable) in their inter-

Table 1: Stable binding of Ran with RCC1 regulates chromatin binding of RCC1 through the N-terminal tail in live cells.

\begin{tabular}{|c|c|c|c|}
\hline Constructs & Mean FRAP half-time \pm SD (s) & Mean mobile fraction \pm SD & $\mathbf{n}$ \\
\hline GFP + mCherry & $0.08 \pm 0.09$ & $0.92 \pm 0.06$ & 19 \\
\hline GFP + mCherry-RanWT & $0.08 \pm 0.08$ & $0.89 \pm 0.04$ & 14 \\
\hline GFP + mCherry-RanT24N & $0.09 \pm 0.09$ & $0.91 \pm 0.06$ & 17 \\
\hline RCC1a-GFP + mCherry & $2.93 \pm 0.56$ & $0.91 \pm 0.05$ & 21 \\
\hline RCC1a-GFP + mCherry-RanWT & $2.85 \pm 0.63$ & $0.91 \pm 0.04$ & 26 \\
\hline RCC1a-GFP + mCherry-RanT24N & $3.69 \pm 0.58$ & $0.87 \pm 0.05$ & 21 \\
\hline$\triangle 27 \mathrm{RCC1}-\mathrm{GFP}+$ mCherry & $0.81 \pm 0.21$ & $0.94 \pm 0.05$ & 23 \\
\hline$\Delta 27 R C C 1-G F P+$ mCherry-RanWT & $0.87 \pm 0.27$ & $0.95 \pm 0.04$ & 23 \\
\hline$\triangle 27 R C C 1-G F P+$ mCherry-RanT24N & $0.87 \pm 0.28$ & $0.94 \pm 0.04$ & 26 \\
\hline RCC1aK4Q-GFP + mCherry & $2.19 \pm 0.44$ & $0.90 \pm 0.06$ & 27 \\
\hline RCC1aK4Q-GFP + mCherry-RanWT & $1.82 \pm 0.54$ & $0.90 \pm 0.06$ & 26 \\
\hline RCC1aK4Q-GFP + mCherry-RanT24N & $2.19 \pm 0.63$ & $0.90 \pm 0.07$ & 30 \\
\hline RCC1aD182A-GFP + mCherry & $1.51 \pm 0.39$ & $0.93 \pm 0.04$ & 20 \\
\hline RCC1aD182A-GFP + mCherry-RanWT & $1.30 \pm 0.54$ & $0.94 \pm 0.05$ & 10 \\
\hline $\mathrm{RCC1aD182A-GFP}+$ mCherry-RanT24N & $2.63 \pm 0.62$ & $0.87 \pm 0.05$ & 12 \\
\hline
\end{tabular}

Fluorescence recovery after photobleaching (FRAP) data derived from live U2OS cells co-expressing GFP, RCC1a-GFP, $\triangle 27 R C C 1-G F P, R C C 1$ a K4QGFP, RCC1aD182A. The table summarises the mean +/- standard deviation (SD) of FRAP half-time and mobile fraction of each of the GFP fusion constructs in the nuclei of cells that also expressed mCherry, mCherry-RanWT or mCherry-RanT24N. The number of cells used in each case is given (n). 
actions with chromatin than the equivalent $\mathrm{C}$-terminal GFP tagged proteins (Additional file 2 Figure S2).

\section{a-N-terminal methylation of RCC1a is required for the stabilization of its interaction with chromatin by $\operatorname{Ran}^{\mathrm{T} 24 \mathrm{~N}}$}

$\mathrm{RCC} 1 \alpha$ is $\alpha$-N-terminally methylated throughout the cell cycle and this modification promotes the localisation of RCC $1 \alpha$ to mitotic chromosomes [18]. To test whether $\alpha$ $\mathrm{N}$-terminal methylation is important for dynamic association of RCC1 with interphase chromatin, we made a mutant of RCC1 $\alpha$ (K4Q), which prevents methylation [18]. As expected, $\alpha-\mathrm{N}$-terminal methylation was completely blocked in $\mathrm{RCC} 1 \alpha^{\mathrm{K} 4 \mathrm{Q}-\mathrm{GFP}}$ and was also absent in $\Delta 27 \mathrm{RCC} 1-\mathrm{GFP}$ but was present in RCC1 $\alpha$-GFP, as well as

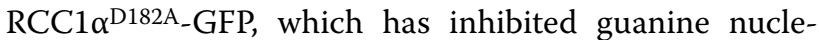
otide exchange activity [12] (Additional file 3 Figure S3). The mobility of RCC $1 \alpha^{\mathrm{K} 4 \mathrm{Q}-\mathrm{GFP}}$ on interphase chromatin was significantly increased $\left(t_{1 / 2}\right.$ decreased) compared to that of wild-type RCC1 $\alpha$-GFP (Table 1, Figure 3) (p < $0.001)$. RCC $1 \alpha^{\mathrm{K} 4 \mathrm{Q}-\mathrm{GFP}}$ also did not associate with chromatin more stably in cells co-expressing $\operatorname{Ran}^{\mathrm{T} 24 \mathrm{~N}}$, in contrast to wild type RCC $1 \alpha-G F P$. Thus, $\alpha$-N-terminal methylation of $\mathrm{RCC} 1 \alpha$ plays a role in its dynamic interaction with interphase chromatin and this modification is important for stabilisation of the interaction with chromatin by $\operatorname{Ran}^{\mathrm{T} 24 \mathrm{~N}}$.

\section{a-N-terminal methylation of RCC1a is not regulated by $\operatorname{Ran}^{\mathrm{T} 24 \mathrm{~N}}$}

We tested further the relationship between the $\alpha$-N-terminal methylation of $\mathrm{RCC} 1 \alpha$ and stabilising effect of $\operatorname{Ran}^{\mathrm{T} 24 \mathrm{~N}}$ on the interaction of $\mathrm{RCC} 1 \alpha$ with chromatin. We found that the co-expression of mCherry-RanWT or mCherry-Ran ${ }^{\mathrm{T} 24 \mathrm{~N}}$ in cells did not affect the $\alpha-\mathrm{N}$-terminal methylation of RCC $1 \alpha-$ GFP or endogenous RCC1 when compared to the co-expression of mCherry alone (Figure 4). Therefore, $\operatorname{Ran}^{\mathrm{T} 24 \mathrm{~N}}$ does not stabilise the interaction of $\mathrm{RCC} 1 \alpha$ with chromatin by inducing the $\alpha$-N-terminal methylation of RCC1 $\alpha$. Conversely, immunoprecipitation of $\mathrm{RCC} 1 \alpha-\mathrm{GFP}$ and $\mathrm{RCC} 1 \alpha^{\mathrm{K} 4 \mathrm{Q}-\mathrm{GFP}}$ in the presence of EDTA, which chelates $\mathrm{Mg}^{2+}$ and releases nucleotides from Ran to form apoRan [27], showed that endogenous Ran formed a stable complex with both RCC $1 \alpha-$ GFP and RCC $1 \alpha^{\mathrm{K} 4 \mathrm{Q}}-\mathrm{GFP}$ (Figure 5). Furthermore, removal of the $\mathrm{N}$-terminal tail did not affect the association of apoRan with $\mathrm{RCC} 1 \alpha$ under these conditions, showing that the methylated N-terminal tail is not required for binding to apoRan. These data indicate that the $\alpha$ - $\mathrm{N}$-terminal methylation of RCC $1 \alpha$ does not affect its association with apoRan. Thus, the $\alpha-\mathrm{N}$-terminal methylation of RCC $1 \alpha$ and the association of the protein with Ran appear to be independent events.

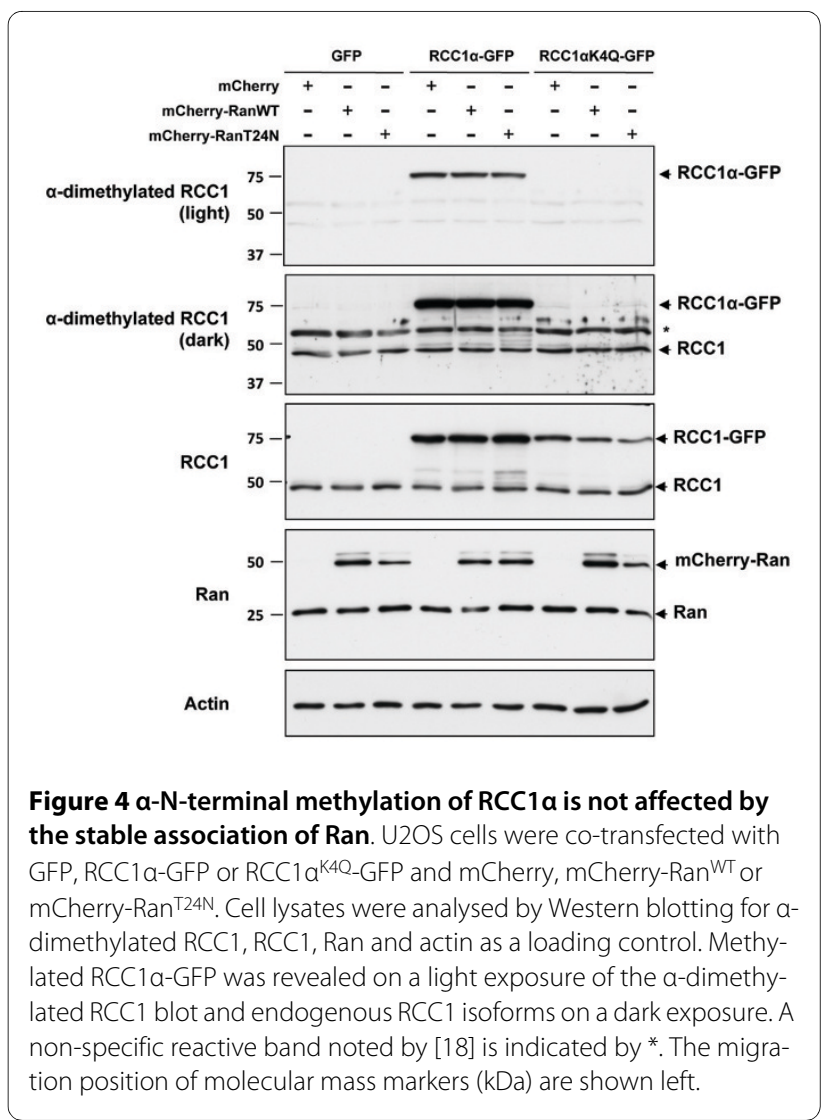

Inhibition of the GEF activity of RCC1 a partially destabilises its interaction with chromatin but does not prevent a stabilising effect of Ran T24N

Mutation of aspartate 182 of RCC1 $\alpha$ to alanine (D182A) strongly reduces $\mathrm{k}_{\mathrm{cat}}$ of its guanine nucleotide exchange activity towards Ran [12]. Aspartate 182 does not interact with Ran directly but rather forms an intramolecular hydrogen bond that stabilises the interaction of arginine 147 of RCC1 $\alpha$ with the Ran P loop [11]. However, the D182A mutant of RCC1 $\alpha$ has been found to disrupt mitosis $[5,18]$ and does not rescue the effects of the mislocalisation of RCC1 $\alpha$ through removal of the $\mathrm{N}$-terminal tail [5]. These effects suggest that the D182A mutant has a dominant effect on the Ran system in cells and is not simply neutral.

In agreement with previous results using $\mathrm{RCC} 1 \alpha$ tagged at the N-terminal with GFP [19] we found that interaction of $\mathrm{RCC} 1 \alpha^{\mathrm{D} 182 \mathrm{~A}}$-GFP with chromatin was more dynamic than the wild-type RCC $1 \alpha-G F P$, showing that this mutation decreased the stability of the interaction of RCC1 $\alpha$ with chromatin (Figure 3). This suggests that the exchange activity of RCC1 promotes its interaction with chromatin. Nevertheless, we found that coexpression of mCherry-Ran ${ }^{\mathrm{T} 24 \mathrm{~N}}$ stabilised the interaction of RCC $1 \alpha^{\mathrm{D} 182 \mathrm{~A}}-\mathrm{GFP}$ with chromatin in live cells. Consis- 


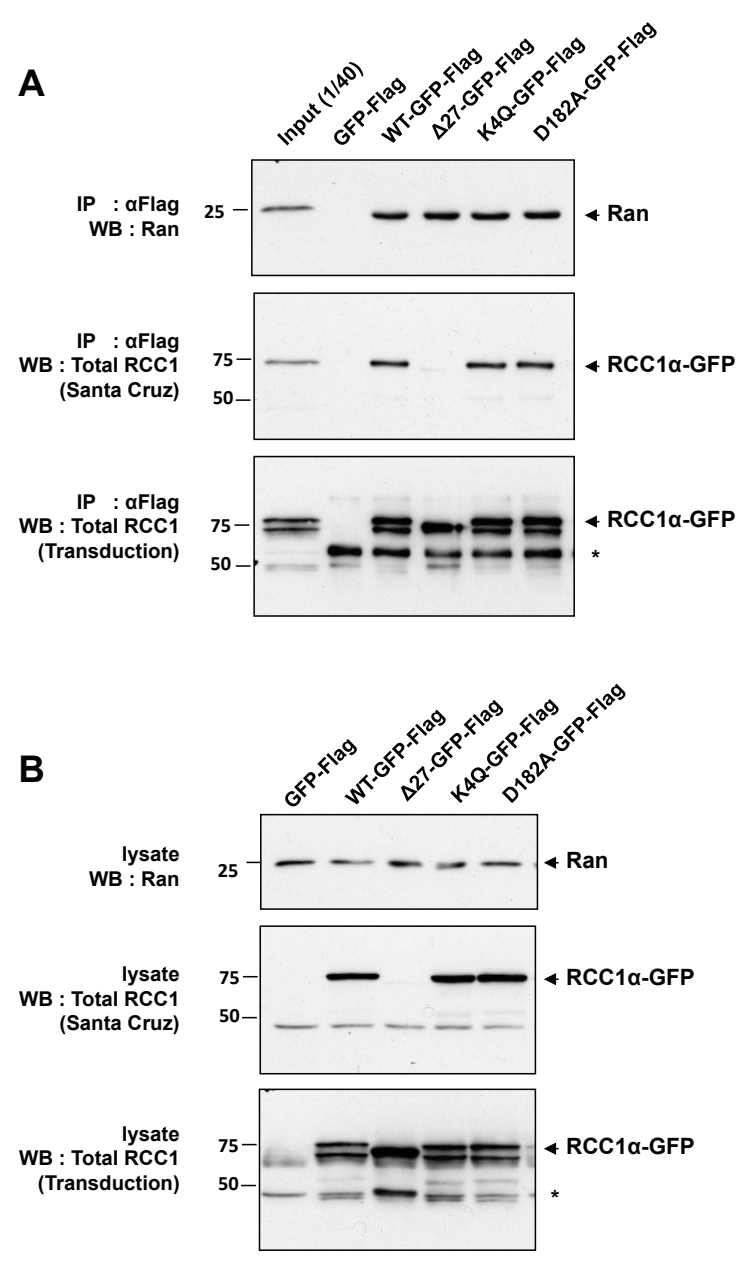

Figure 5 Mutations of RCC1 that remove the N-terminal tail, block N-terminal methylation or inhibit GEF activity do not prevent its interaction with apoRan. RCC1 a wild-type (WT) and mutants $(\triangle 27, \mathrm{~K} 4 \mathrm{Q}$ and D182A) fused at the C-terminal with GFP-FLAG were expressed in U2OS cells (A) and immunoprecipitated with a-FLAG agarose beads (B). RCC1a-GFP-FLAG proteins were analysed by Western blotting using antibodies from Santa Cruz Biotechnology (sc-1162; does not recognize $\triangle 27 R C C 1$ ) (middle panel) and Transduction Laboratories (R35420) (bottom panel). The Transduction antibody detects both full-length RCC1a-GFP-FLAG and a lower band that is not detected by the Santa Cruz antibody. The latter therefore probably represents a truncated form lacking the N-terminal tail of RCC1a. A prominent non-specific band reacting with the Transduction antibody is indicated by *. Endogenous Ran present in the cell lysates (A) or coimmunoprecipitated with RCC1 a-GFP-FLAG proteins (B) was detected by a specific antibody (top panel).

tent with this observation, $\mathrm{RCC} 1 \alpha^{\mathrm{D} 182 \mathrm{~A}}$-GFP tagged with

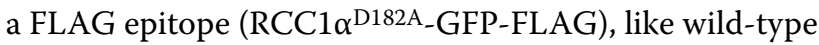
RCC1 $\alpha$-GFP-FLAG and RCC $1 \alpha^{\mathrm{K} 4 \mathrm{Q}-G F P-F L A G, ~ p r e c i p i-~}$ tated Ran from cells under $\mathrm{Mg}^{2+}$-chelated conditions, i.e. when a stable complex between RCC1 $\alpha$ and apoRan is formed (Figure 5). Therefore, we show that D182A mutant of $\mathrm{RCC} 1 \alpha$ still interacts with apoRan and this binary complex is sufficiently stable in the absence of guanine nucleotide binding for it to be co-precipitated. The stabilising effect of $\operatorname{Ran}^{\mathrm{T} 24 \mathrm{~N}}$ on the interaction of $\mathrm{RCC} 1 \alpha^{\mathrm{D} 182 \mathrm{~A}}$ with chromatin indicates that the D182A mutation does not prevent the allosteric effect of Ran on the $\mathrm{N}$-terminal tail of $\mathrm{RCC} 1 \alpha$.

\section{Discussion}

In vitro, the stable association of nucleotide-free Ran with $\mathrm{RCC} 1 \alpha$ produces a conformational change in the $\mathrm{N}$ terminal tail that promotes binding to DNA and reduces affinity for core histones [13]. In cells, however, the interaction of RCC1 isoforms with chromatin is highly dynamic and is affected by post-translational modification of the tail. Our results confirm that this dynamic interaction is regulated by the association of Ran with

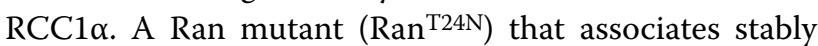
with $\mathrm{RCC} 1 \alpha$ in a binary complex strongly reduces the mobility of RCC1 $\alpha$ on chromatin, and this effect requires the methylated $\mathrm{N}$-terminal tail of $\mathrm{RCC} 1 \alpha$.

Our results are consistent with a model in which apoRan (or $\operatorname{Ran}^{\mathrm{T} 24 \mathrm{~N}}$ ) associates with the core domain of $\mathrm{RCC} 1 \alpha$ and induces allosterically a conformational change in the $\mathrm{N}$-terminal tail that stabilises interaction with chromatin. $\mathrm{RCC} 1 \alpha$ associates weakly with chromatin through its core domain, possibly through interactions with core histones [13]. The interaction of nucleotide-bound Ran with RCC1 $\alpha$ releases the nucleotide from Ran, forming a transient binary complex in which a conformational change in $\mathrm{RCC} 1 \alpha$ exposes its $\mathrm{N}$ terminal domain and stabilises its interaction with chromatin (Figure 6), possibly through direct interaction with DNA, although this remains to be confirmed in vivo.

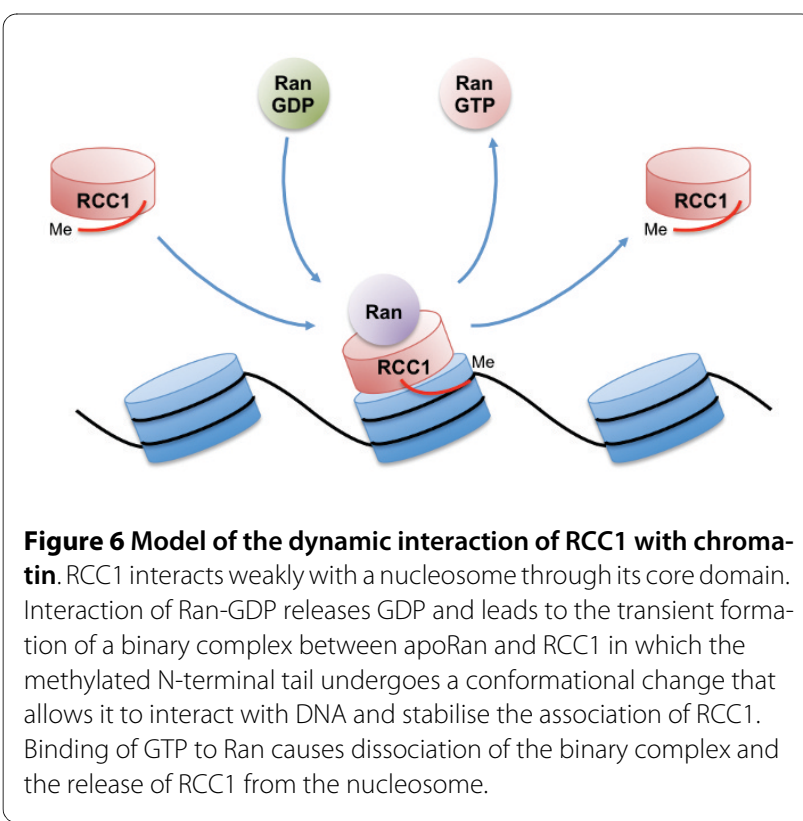


Other isoforms of RCC1 differ in the length of their $\mathrm{N}$ terminal tails and in the turnover rate of their dynamic interactions with chromatin in cells [21], but it is likely that all isoforms interact with chromatin through a similar mechanism, albeit with differing affinities depending on the composition of the tail.

Our results with the D182A mutant of $\mathrm{RCC} 1 \alpha$, which inhibits guanine nucleotide exchange activity, are consistent with a model in which the dynamic interaction of $\mathrm{RCC} 1 \alpha$ with chromatin is linked to its interaction with Ran. Previous experiments by Azuma and colleagues [12] using purified proteins have shown that the D182A mutant of RCC $1 \alpha$ forms a binary complex with apoRan at a reduced rate compared to wild-type $\mathrm{RCC} 1 \alpha$, and the apoRan-RCC $1 \alpha^{\mathrm{D} 182 \mathrm{~A}}$ complex dissociates slowly even in the absence of free guanine nucleotide whereas the wildtype binary complex is stable. However, addition of guanine nucleotide causes rapid dissociation of both the RCC1 $\alpha$ wild-type and D182A binary complexes [12]. Therefore the T24N mutation of Ran (which prevents nucleotide binding) can be envisaged to stabilise even the interaction with $\mathrm{RCC} 1 \alpha^{\mathrm{D} 182 \mathrm{~A}}$ to an extent and thereby promote the chromatin-binding conformation of this binary complex in cells. Nevertheless, the combination of reduced association rate of apoRan with $\mathrm{RCC} 1 \alpha^{\mathrm{D} 182 \mathrm{~A}}$ and likely increased dissociation rate of apoRan from $\mathrm{RCC} 1 \alpha^{\mathrm{D} 182 \mathrm{~A}}$ results in the interaction of $\mathrm{RCC} 1 \alpha^{\mathrm{D} 182 \mathrm{~A}}$ with chromatin being more dynamic than wild-type RCC1 $\alpha$ even when $\operatorname{Ran}^{\mathrm{T} 24 \mathrm{~N}}$ is present.

In agreement with the partial stabilising effect of $\operatorname{Ran}^{\mathrm{T} 24 \mathrm{~N}}$ on the interaction of $\mathrm{RCC} 1 \alpha^{\mathrm{D} 182 \mathrm{~A}}$ with chromatin, we have also observed a complex formed between $\mathrm{RCC} 1 \alpha^{\mathrm{D} 182 \mathrm{~A}}$ and $\mathrm{Ran}$ under $\mathrm{Mg}^{2+}$-chelating conditions, which promote the nucleotide-free form of Ran (apoRan). This suggests that the apoRan-RCC1 $\alpha^{\mathrm{D} 182 \mathrm{~A}}$ binary complex might be more stable in cells than was predicted. Indeed, the stability of the wild-type apoRan-RCC1 $\alpha$ binary complex when it interacts with chromatin is not yet known. A recent study of the interaction of $\mathrm{RCC} 1 \alpha$ with nucleosomes in solution has suggested the interesting possibility that Ran could interact with $\mathrm{RCC} 1 \alpha$ on chromatin in a different conformation from the crystallised binary complex [28], although the formation of such a distinct complex under cellular conditions remains to be confirmed. Whatever its conformation, the assembly of a complex between Ran and $\mathrm{RCC} 1 \alpha^{\mathrm{D} 182 \mathrm{~A}}$ that does not result in the efficient loading of Ran with GTP provides an explanation for the inhibitory effect of this mutant on mitosis [5,18].

The precise relationship between the dynamics of the interaction of $\mathrm{RCC} 1 \alpha$ with chromatin and its guanine nucleotide exchange activity is, however, not yet certain. In one model, they are tightly coupled: binding of nucle- otide to apoRan causes Ran-GTP (or Ran-GDP) to dissociate from $\mathrm{RCC} 1 \alpha$, then $\mathrm{RCC} 1 \alpha$ is released from chromatin and the $\mathrm{N}$-terminal tail folds back against the core domain (Figure 6). Although $\mathrm{RCC} 1 \alpha$ can catalyse the reaction equally well from GDP to GTP and vice versa, the presence of accessory factors and the higher concentration of GTP than GDP in cells results in net loading of Ran with GTP. Alternatively, RCC $1 \alpha$ could remain associated with chromatin for more than one guanine nucleotide exchange reaction if its dissociation from chromatin is slower than the release of Ran.

The conformational change in the N-terminal tail and/ or its interaction with chromatin may be regulated by post-translational mechanisms as well as through the binding of Ran to RCC1 isoforms. Throughout the cell cycle, mono-, di- or tri-methylation of the $\alpha$-amino group promotes the interaction with chromatin [18], whereas in mitosis, phosphorylation of serines 2 and 11 makes the interaction more dynamic $[19,20]$. We have found that $\alpha$ $\mathrm{N}$-terminal methylation of $\mathrm{RCC} 1 \alpha$ is not affected by its stable association with Ran. We therefore favour the idea that post-translational modification of the N-terminal tail is relatively stable and is not tightly linked to the cycle of association and dissociation of RCC1 with chromatin, but rather alters the equilibrium of this interaction towards association with chromatin.

\section{Conclusions}

Stabilisation of the dynamic interaction of $\mathrm{RCC} 1 \alpha$ with chromatin by Ran requires the $\alpha-\mathrm{N}$-methylated $\mathrm{N}$-terminal tail of RCC1 $\alpha$. This is consistent with the transient formation of the binary complex between RCC1 and Ran in which $\mathrm{N}$-terminal tail of RCC1 undergoes a conformational change that allows it to interact more stably with chromatin. The coupling between the association of RCC1 with Ran and its interaction with chromatin provides an unusual mechanism to localise the generation of Ran-GTP.

\section{Methods}

\section{Tissue culture}

Human HeLa and U2OS cells obtained from Cancer Research UK London Institute were cultured in Dulbecco's Modified Eagle Medium (DMEM) (Invitrogen) supplemented with 10\% Foetal Bovine Serum (Biosera), 50 units/ml Penicillin G (Invitrogen), $50 \mu \mathrm{g} / \mathrm{ml}$ Streptomycin (Invitrogen) and $2 \mathrm{mM} \mathrm{L-glutamine} \mathrm{(Invitrogen).}$ Cells were grown at $37^{\circ} \mathrm{C}$ in $5 \% \mathrm{CO}_{2}$ incubator. For FRAP experiments, cells were transfected with plasmids encoding GFP fusion constructs using Fugene HD transfection reagent following manufacturer's protocol. 24 hours posttransfection, media were replaced with phenol red-free DMEM (Invitrogen) and subject to imaging. 


\section{Generation of GFP fusion constructs}

All of the RCC1 constructs used in this study are derived from the major $\alpha$ isoform of human RCC1 [21]. To generate the GFP fusion $\mathrm{RCC} 1 \alpha$ constructs, $\mathrm{RCC} 1 \alpha \mathrm{N}$-terminal region (amino acids 1-27) was amplified and inserted into EcoRI and Sall sites of pEGFP-N1 (Clonetech) to generate the $\mathrm{C}$-terminally GFP tagged vector expressing $\mathrm{N}$-terminal tail of RCC1 $\alpha$. Site directed mutagenesis was also used to introduce SV40 nuclear localization signal (NLS) into PstI site of both pEGFP-N1 vectors. The fulllength sequence of RCC1 downstream of codon 27 (RCC1 127$)$ was amplified and inserted into KpnI and BamHI sites or KpnI and AgeI sites of pEGFP-C3 and pEGFP-N1 vectors to generate GFP- $227 R C C 1$ and $\triangle 27 R C C 1-G F P$ fusion constructs, respectively. Following, site-directed mutagenesis was performed to introduce SV40 NLS into Pst I site of both vectors to generate GFP-SV40 NLS- $\triangle 27$ RCC1 (GFP-SVRCC1) and SV40 NLS- 2 27RCC1-GFP (SVRCC1-GFP), respectively.

Ran wild-type and T24N mutant cDNA were amplified from GFP-Ran ${ }^{\mathrm{WT}}$ and GFP-Ran ${ }^{\mathrm{T} 24 \mathrm{~N}}$ [26] and inserted into EcoRI and BamHI sites of an mCherry vector (a kind gift of Professor Angus Lamond, University of Dundee) to generate mCherry fusion constructs. The FLAG tag was inserted before the stop codon of GFP in pEGFP-N1 by site-directed mutagenesis. Sequences of the constructs made were verified by direct sequencing to ensure that no mutations were introduced.

\section{Fluorescent recovery after photobleaching (FRAP)}

U2OS and HeLa cells were cultured in Phenol-red free DMEM (Invitrogen) on glass bottom dishes $(35 \times 22 \mathrm{~mm})$ (Intracel). All constructs were fused with GFP at either Cterminus or $\mathrm{N}$-terminus and expressed in Hela cells or U2OS cells where indicated. Fluorescence recovery after photobleaching (FRAP) experiments were performed on a DeltaVision Spectris microscopy workstation based on an Olympus IX70 inverted widefield deconvolution microsope equipped with QLM laser module. Live cells expressing GFP fusion and/or mCherry fusion constructs where indicated were scanned 3 times and GFP was photobleached using $488 \mathrm{~nm}$ laser at a defined spot on chromatin. Following, single images $(512 \times 512$ pixels $)$ were captured with the exposure time of $100 \mathrm{~ms}$ for 50 time points to monitor the FRAP half-time $\left(t_{1 / 2}\right)$. Data were processed with softWoRx software (Applied Precision).

\section{Western blotting}

Proteins were resolved on SDS-PAGE gels and transferred onto nitrocellulose membrane (Amersham). Membranes were incubated in $5 \%$ dried skimmed milk/PBSTween $(0.1 \%)$ for an hour and then with indicated primary antibodies diluted in 5\% milk/PBS-Tween overnight at $4^{\circ} \mathrm{C}$. Membranes were washed three times in PBS-
Tween. Membranes were incubated with secondary antibodies diluted in 5\% milk/PBS-Tween for an hour at room temperature and then washed three times in PBSTween.

\section{Antibodies}

Goat anti-RCC1 polyclonal antibody (used at 1:1000 dilution) was from Santa Cruz Biotechnology (C-20: sc1162). This antibody is described as having been raised against a C-terminal peptide, although we find that it does not recognize $\mathrm{RCC} 1 \alpha$ lacking the first 27 residues $(\triangle 27 R C C 1)$ (Additional file 3 Figure S3). Mouse antiRCC1 monoclonal antibody from Transduction Laboratories (R35420). Mouse anti-FLAG monoclonal antibody (used at 1:8000 dilutions) and rabbit anti-actin polyclonal antibody (used at 1:5000 dilution) were from Sigma. Rabbit anti- $\alpha$-dimethylated RCC1 polyclonal antibody (used at 1:1000 dilutions) was a kind gift of Dr. Ian Macara (University of Virginia). Goat anti-Ran (C-20: sc-1156) polyclonal antibody (used at 1:1000 dilution) was from Santa Cruz Biotechonology.

\section{FLAG Immunoprecipitation (IP)}

Asynchronous U2OS cells were co-transfected with various RCC1 GFP-FLAG constructs and either mCherry empty vector or mCherry-Ran ${ }^{W T}$ fusion construct using Fugene HD transfection reagent (Roche) following manufacturer's protocol. 24 hours after transfection, cells were lysed in IP buffer (50 mM Tris pH 8.0, 2 mM EDTA pH 8.0, $150 \mathrm{mM}$ sodium chloride, $50 \mathrm{mM}$ sodium fluoride, 5 $\mathrm{mM} \beta$-glycerophosphate, $1 \mathrm{mM}$ sodium orthovanadate, $1 \%$ Triton $\mathrm{X}-100$ ) supplemented with protease inhibitors and $1 \mathrm{mM}$ okadaic acid. Lysates were left on ice for 20 minutes and spun down at $4^{\circ} \mathrm{C}$ for 20 minutes. FLAG beads (Sigma) were pre-washed 3 times in IP buffer. $1 \mathrm{mg}$ lysates were then added to washed beads and incubated on wheel at $4^{\circ} \mathrm{C}$ for 90 minutes. Beads were washed with IP buffer 3 times. The immune complex was eluted with SDS loading buffer containing 5\% $\beta$-mercaptoethanol. Samples were run on SDS-PAGE gel and blotted with indicated antibodies.

\section{Statistical analysis}

Differences in mean of FRAP half-time $\left(t_{1 / 2}\right)$ was tested using Student's $t$-test (to compare means of 2 samples) and ANOVA test (to compare means of more than 2 samples) using statistics software (SPSS Inc.).

\section{Additional material}

Additional file 1 Figure S1. Fluorescence recovery after photobleaching (FRAP) of RCC $1 a$ and mutants fused to GFP at the N-terminus or the C-terminus.

Additional file $\mathbf{2}$ Figure S2. Fluorescence recovery after photobleaching (FRAP) showing stabilisation of the interaction of GFP-RCC1 a (N-terminal GFP) with chromatin by Ran ${ }^{T 24 N}$ 
Additional file 3 Figure S3. Deletion of the $\mathrm{N}$-terminal tail and mutation of lysine 4 (K4Q) abolishes methylation of RCC1a. Western blot showing the a-N-dimethylation of RCC1 a, $\triangle 27 R C C 1, R C C 1 a^{K 4 Q}, R C C 1 a^{D 182 A}$ and RCC $1 a^{\text {S11A }}$

\section{Authors' contributions}

EH generated and analysed the data. FEH made GFP-fusion constructs and provided preliminary data. HSS provided supporting data and participated in the design of the study. PRC conceived of the study and participated in its design. EH wrote the manuscript together with PRC. All authors read and approved the final manuscript.

\section{Acknowledgements}

We thank Sam Swift for help and advice with photobleaching experiments and Angus Lamond for the mCherry vector (both University of Dundee). We also thank lan Macara (University of Virginia) for the kind gift of the antibody that detects dimethylated RCC1. The work was supported by a studentship of the Royal Thai Government (EH), a studentship of the Medical Research Council (FEH), the Biotechnology and Biological Sciences Research Council (HSS) and Tenovus Scotland. PRC was recipient of a Royal Society-Wolfson Research Merit Award.

\section{Author Details}

${ }^{1}$ Biomedical Research Institute, School of Medicine, College of Medicine, Dentistry and Nursing, University of Dundee, Ninewells Hospital and Medical School, Dundee DD1 9SY, UK and 2The Physiological Laboratory, School of Biomedical Sciences, University of Liverpool, Liverpool L69 3BX, UK

Received: 21 December 2009 Accepted: 21 June 2010

Published: 21 June 2010

\section{References}

1. Clarke PR, Zhang C: Spatial and temporal coordination of mitosis by Ran GTPase. Nat Rev Mol Cell Biol 2008, 9:464-477.

2. Ohtsubo M, Kai R, Furuno N, Sekiguchi T, Sekiguchi M, Hayashida H, Kuma K, Miyata T, Fukushige S, Murotsu T, et al.: Isolation and characterization of the active cDNA of the human cell cycle gene (RCC1) involved in the regulation of onset of chromosome condensation. Genes Dev 1987 1:585-593.

3. Bischoff FR, Ponstingl $\mathrm{H}$ : Catalysis of guanine nucleotide exchange on Ran by the mitotic regulator RCC1. Nature 1991, 354:80-82.

4. Ohtsubo M, Okazaki $\mathrm{H}$, Nishimoto $\mathrm{T}$ : The RCC1 protein, a regulator for the onset of chromosome condensation locates in the nucleus and binds to DNA. J Cell Biol 1989, 109:1389-1397.

5. Moore W, Zhang C, Clarke PR: Targeting of RCC1 to chromosomes is required for proper mitotic spindle assembly in human cells. Curr Biol 2002, 12:1442-1447.

6. Bischoff FR, Klebe C, Kretschmer J, Wittinghofer A, Ponstingl H: RanGAP1 induces GTPase activity of nuclear Ras-related Ran. Proc Natl Acad Sci USA 1994, 91:2587-2591.

7. Kalab P, Pralle A, Isacoff EY, Heald R, Weis K: Analysis of a RanGTPregulated gradient in mitotic somatic cells. Nature 2006, 440:697-701.

8. Görlich D, Mattaj IW: Nucleocytoplasmic transport. Science 1996 271:1513-1518.

9. Hetzer M, Gruss OJ, Mattaj IW: The Ran GTPase as a marker of chromosome position in spindle formation and nuclear envelope assembly. Nat Cell Biol 2002, 4:E177-184.

10. Renault L, Nassar N, Vetter I, Becker J, Klebe C, Roth M, Wittinghofer A: The 1.7 A crystal structure of the regulator of chromosome condensation (RCC1) reveals a seven-bladed propeller. Nature 1998, 392:97-101.

11. Renault L, Kuhlmann J, Henkel A, Wittinghofer A: Structural basis for guanine nucleotide exchange on Ran by the regulator of chromosome condensation (RCC1). Cell 2001, 105:245-255.

12. Azuma Y, Renault L, Garcia-Ranea JA, Valencia A, Nishimoto T, Wittinghofer A: Model of the Ran-RCC1 interaction using biochemical and docking experiments. J Mol Biol 1999, 289:1119-1130.

13. Hao Y, Macara IG: Regulation of chromatin binding by a conformational switch in the tail of the Ran exchange factor RCC1. J Cell Biol 2008, 182:827-836.
14. Nemergut ME, Mizzen CA, Stukenberg T, Allis CD, Macara IG: Chromatin docking and exchange activity enhancement of RCC 1 by histones $\mathrm{H} 2 \mathrm{~A}$ and H2B. Science 2001, 292:1540-1543.

15. Nemergut ME, Macara IG: Nuclear import of the Ran exchange factor, RCC 1 , is mediated by at least two distinct mechanisms. J Cell Biol 2000, 149:835-850.

16. Talcott B, Moore MS: The nuclear import of RCC1 requires a specific nuclear localization sequence receptor, karyopherin alpha3/Qip. J Biol Chem 2000, 275:10099-10104

17. Seino H, Hisamoto N, Uzawa S, Sekiguchi T, Nishimoto T: DNA-binding domain of RCC1 protein is not essential for coupling mitosis with DNA replication. J Cell Sci 1992, 102:393-400.

18. Chen T, Muratore TL, Schaner-Tooley CE, Shabanowitz J, Hunt DF, Macara IG: N-terminal alpha-methylation of RCC1 is necessary for stable chromatin association and normal mitosis. Nat Cell Biol 2007, 9:596-603.

19. Hutchins JR, Moore WJ, Hood FE, Wilson JS, Andrews PD, Swedlow JR, Clarke PR: Phosphorylation regulates the dynamic interaction of RCC1 with chromosomes during mitosis. Curr Biol 2004, 14:1099-1104.

20. Li HY, Zheng Y: Phosphorylation of RCC1 in mitosis is essential for producing a high RanGTP concentration on chromosomes and for spindle assembly in mammalian cells. Genes Dev 2004, 18:512-527.

21. Hood FE, Clarke PR: RCC1 isoforms differ in their affinity for chromatin, molecular interactions and regulation by phosphorylation. J Cell SCi 2007, 120:3436-3445.

22. Li HY, Wirtz D, Zheng Y: A mechanism of coupling RCC1 mobility to RanGTP production on the chromatin in vivo. J Cell Biol 2003, 160:635-644.

23. Cushman I, Stenoien D, Moore MS: The dynamic association of RCC1 with chromatin is modulated by Ran-dependent nuclear transport. Mol Biol Cell 2004, 15:245-255.

24. Dasso M, Seki T, Azuma Y, Ohba T, Nishimoto T: A mutant form of the Ran/TC4 protein disrupts nuclear function in Xenopus laevis egg extracts by inhibiting the RCC1 protein, a regulator of chromosome condensation. EMBO J 1994, 13:5732-5744.

25. Klebe C, Bischoff FR, Ponstingl H, Wittinghofer A: Interaction of the nuclear GTP-binding protein Ran with its regulatory proteins RCC1 and RanGAP1. Biochemistry 1995, 34:639-647.

26. Hutchins JR, Moore WJ, Clarke PR: Dynamic localisation of Ran GTPase during the cell cycle. BMC Cell Biol 2009, 10:66

27. Bischoff FR, Krebber $\mathrm{H}$, Smirnova $\mathrm{E}$, Dong W, Ponstingl $\mathrm{H}$ : Co-activation of RanGTPase and inhibition of GTP dissociation by Ran-GTP binding protein RanBP1. EMBO J 1995, 14:705-715.

28. England JR, Huang J, Jennings MJ, Makde RD, Tan S: RCC1 Uses a Conformationally Diverse Loop Region to Interact with the Nucleosome: A Model for the RCC1-Nucleosome Complex. J Mol Biol 2010, 398:518-529.

doi: $10.1186 / 1471-2121-11-43$

Cite this article as: Hitakomate et al., The methylated N-terminal tail of RCC1 is required for stabilisation of its interaction with chromatin by Ran in live cells BMC Cell Biology 2010, 11:43

\section{Submit your next manuscript to BioMed Centra and take full advantage of:}

- Convenient online submission

- Thorough peer review

- No space constraints or color figure charges

- Immediate publication on acceptance

- Inclusion in PubMed, CAS, Scopus and Google Scholar

- Research which is freely available for redistribution 